\title{
Peculiarities of the Rice Statistical Distribution: Mathematical Substantiation
}

\author{
Tatiana Yakovleva \\ Federal Research Center "Computer Science and Control” of the Russian Academy of Sciences, Moscow, Russia \\ Email address: \\ tan-ya $a$ bk.ru

\section{To cite this article:} \\ Tatiana Yakovleva. Peculiarities of the Rice Statistical Distribution: Mathematical Substantiation. Applied and Computational Mathematics. \\ Vol. 7, No. 4, 2018, pp. 188-196. doi: 10.11648/j.acm.20180704.12
}

Received: June 7, 2018; Accepted: August 15, 2018; Published: September 18, 2018

\begin{abstract}
The Rice statistical distribution has recently become a subject of increasing scientific interest due to its wide applicability in various fields of science and technology, such as the magnetic-resonance visualization and ultrasound diagnostics in medicine, the radio and radar signals' analysis and processing, the optical measurements, etc. The common feature of the tasks that are adequately described by the Rician statistical model consists in the fact that the value to be measured and analyzed is an amplitude, or an envelope of the output signal which is composed as a sum of the initially determined informative component and a random noise component being formed by many independent normally-distributed summands. The efficient reconstruction of the Rician signal's informative component against the noise background is shown to be achieved only on the basis of joint determination of both a priori unknown Rician parameters. The Rice statistical distribution possesses some peculiarities that allow solving rather complicated tasks connected with the stochastic data processing. The paper considers the issues of the strict mathematical substantiations of the Rice distribution properties that are meaningful for its efficient application, namely: the Rician likelihood function features and the stable character of the Rice distribution. There are provided the rigorous proofs of the mentioned properties.
\end{abstract}

Keywords: Rice Distribution, Probability Density, Likelihood Function, Data Processing

\section{Introduction}

Modern science is characterized by dynamic development of new mathematical techniques that provide enhancing the efficiency of data analyzing and processing. As a rule the data being analyzed are of random, stochastic character. That's why the techniques being used at solving the tasks of data analysis should take into account the peculiar features of the statistical distribution of the data to be analyzed.

A recently increasing interest to the problem of the Rician signals analysis [1-5] is connected with a wide circle of scientific and technological tasks which are adequately described by the Rician statistical model. The common feature of these tasks consists in the following: the value to be analyzed is an amplitude, or an envelope of the output signal that is composed by a sum of initially determined signal and a random noise while the noise being formed by many independent normally-distributed summands with zero mean, [6]. Such a random variable is known to obey to the Rice statistical distribution. The range of the problems that are mathematically described by the Rice distribution includes the tasks of magnetic-resonance visualization [7, 8], the radio signals reception and processing, the tasks of the radar signals analysis [9], as well as the analysis of the sonar signals, the measurements of the optical medium's properties by analyzing the reflected wave $[10,11]$, etc.

Taking into account the physical meaning of the parameters of Rice statistical distribution one can conclude that the task of the useful, informative signal reconstruction against the noise background at Rician data analysis is equivalent to the task of the both Rician parameters estimation.

The paper presents the theoretical study of the Rice statistical distribution's peculiarities that allow efficient processing of Rician data by means of the so-called two-parameter analysis. Such an analysis provides the possibility of joint signal and noise calculation by measuring the sum signal. 


\section{Relation to Prior Art}

The Rice distribution was first formulated by Stephan Rice [1] as an extension of the Rayleigh distribution well known from the classical probability theory on the case of nonzero amplitude of the initial signal. The Rice statistical model [1] adequately describes the processes of various physical nature, when the noise is formed by summing a big number of normally-distributed components while the analyzed value is just an amplitude of the signal.

A significant interest to solving a task of joint estimation of both parameters of the Rice distribution has appeared in 60-th years of the 20th century due to understanding that in conditions of Rice distribution only the knowledge of both Rician parameters allows efficient reconstructing the initial required signal against the noise background. In paper [2] the two-parameter task was considered applicably to radar signals' analysis. However, this task solving has appeared to be conjugated with considerable difficulties of both the theoretical and the computational character. Later the simplified methods of the Rician data analysis have been elaborated in the conditions of the so-called one-parameter approximation consisting in estimating only one of the two unknown parameters - the signal value, in supposition that the second parameter - the noise dispersion - is known a priori, [3] and [4].

However, in practice the condition when the Gaussian noise dispersion is known a priori never takes place and such a supposition is a severe restriction of the one-parameter approach's applicability.

Therefore, the theoretical problem of joint estimation of both Rician parameters without any a priori conditions has remained unsolved for a few decades, since the 60 -th years of the $20^{\text {th }}$ century. The strict mathematical grounds for the theory of the two-parameter approach to solving the task of Rician data analysis have been first provided in [5, 12-15].

The present paper is directed onto the detailed study of the Rice statistical distribution's peculiarities and provides their mathematical substantiation.

\section{Theoretical Basics of the Rician Data Analysis}

The Rician distribution is known to describe an amplitude of the random variable, formed by summing an initially determined complex signal and the Gaussian noise distorting this signal.

Let $A$ be a determined value that characterizes the physical process to be considered. This value is inevitably distorted by the Gaussian noise created by a great number of independent noise components, while the measured and analyzed value is an amplitude, or the envelope of the resulting signal. The Gaussian noise distorting the initial determined signal is characterized by a zero mean value and a dispersion $\sigma^{2}$. Thus the task consists in the analysis of the signal's amplitude $x=\sqrt{x_{\mathrm{Re}}^{2}+x_{\mathrm{Im}}^{2}}$.
The real $x_{\mathrm{Re}}$ and imaginary $x_{\mathrm{Im}}$ parts of the complex signal with amplitude $x$ are random Gaussian values with mathematical expectations $\overline{x_{\mathrm{Re}}}$ and $\overline{x_{\mathrm{Im}}}$, satisfying the condition ${\overline{x_{\mathrm{Re}}}}^{2}+{\overline{x_{\mathrm{Im}}}}^{2}=A^{2}$, and dispersion $\sigma^{2}$. The random value $x$ obeys the Rice distribution with parameters $v=A$ and $\sigma$, [1]. Obviously, the value of $x$ belongs to the subset of the not-negative real numbers: $x \in(0, \infty)$. The ratio of the Rician parameters $S N R=v / \sigma$ characterises the signal-to-noise ratio.

So, the Rician random variable $x$ represents the amplitude of the signal with the Gaussian real and imaginary parts. The Rician probability density function is given by the following formula:

$$
P\left(x \mid v, \sigma^{2}\right)=\frac{x}{\sigma^{2}} \cdot \exp \left(-\frac{x^{2}+v^{2}}{2 \sigma^{2}}\right) \cdot I_{0}\left(\frac{x v}{\sigma^{2}}\right),(1)
$$

where $I_{0}$ is the modified Bessel function of the first kind of order zero, [16]. Here and below the following denotations are used: $I_{\alpha}(z)$ is the modified Bessel function of the first kind (or the Infeld function) of the order $\alpha ; x_{i}$ is the signal's value measured as the $i$-th element of a sample; $n$ is the quantity of elements in a sample, called also a sample's length.

The final purpose of the Rician data processing is evidently the evaluation of value $A$ that characterizes the process under the study and coincides with parameter $v$ of the Rice distribution.

The Rician value's mathematical expectation and dispersion are known to be expressed by the following formulas, [17]:

$$
\begin{gathered}
\bar{x}=\sigma \cdot \sqrt{\pi / 2} \cdot L_{1 / 2}\left(-v^{2} / 2 \sigma^{2}\right), \\
\sigma_{x}^{2}=2 \cdot \sigma^{2}+v^{2}-\sigma^{2} \cdot \frac{\pi}{2} \cdot L_{1 / 2}^{2}\left(-v^{2} / 2 \sigma^{2}\right),
\end{gathered}
$$

where function $L_{1 / 2}(z)$ is the Laguerre polynomial.

As one can see the values $\bar{x}$ and $\sigma_{x}^{2}$ do not coincide with the Rician parameters $v$ and $\sigma^{2}$, correspondingly. Nevertheless the both Rician parameters have a certain physical sense, namely: $\sigma^{2}$ is $\mathrm{s}$ dispersion of the Gaussian noise distorting the initial signal, while parameter $v$ coincides with the value of the initially determined signal $v=A$.

From the mathematical peculiarities of the Rician distribution noticed above it follows that the Rician signal's mean value (2) and its dispersion (3) depend on the both Rician parameters: $v$ and $\sigma^{2}$. This means that the efficient reconstruction of the Rician signal against the noise background demands solving the task of joint evaluation of $a$ priori unknown parameters $v$ and $\sigma^{2}$.

The solution of the formulated two-parameter task allows reconstructing the sought for values of the initial, not-noised 
signal $A$, coinciding with parameter $v: A=v$.

\section{Parameters of the Rice Distribution: Physical Meaning and Peculiarities of Estimating}

The foregoing allows substantiating the choice of the Rice statistical model for the joint signal and noise estimation for solving the task in which the analyzed value is an amplitude and the noise obeys to the Gaussian statistics. The Gauss and the Rayleigh distributions can be considered as its special cases at the limits of very high and very low signal-to-noise ratio.

By virtue of the specifics of the Rice distribution the analysis of the Rician data demands the development of special methods and the corresponding mathematical apparatus. It is well known that at the Gaussian data analysis a traditional and efficient filtration tool is the data averaging. However, in contrast to the case of the Gaussian distribution the average value of the Rician signal $\bar{x}$ does not coincide with the value of the sought for useful signal $v$. This is illustrated by Figure 1 where the average value of the Riciansignal $\bar{x}$, depending upon the Rician parameters according to (2), is depicted by the curve line, while the value of the sought for useful signal $v$ is depicted by the straight line outgoing from the origin of coordinates.

The plots in Figure 1 correspond to the constant value of parameter $\sigma: \sigma=1$, so that the points of the abscissa axes correspond to the value of signal-to-noise ratio $S N R=v / \sigma$.

Therefore, if one applies to the Rician data processing the traditional technique of the filtration by means of the data averaging, then within the range of small values of the signal-to-noise ratio the result will be just a leveling of the real signal values. So, the peculiarities of the Rician distribution are first of all determined by the fact that the average value of the Rician signal does not coincide with the initial, un-noised signal. Similarly, the dispersion of the Rician signal does not coincide with the dispersion of Gaussian noise that forms the Rician signal from the initially determined value. The Rician noise non-linearity is also a peculiar feature of the Rice statistical model. These properties are inherent to the Rice distribution and, in contrast to the Gaussian statistical model do not allow analyzing data just by simple averaging. Instead the indicated peculiarities of the Rician random variable predetermine the necessity of the development of special theoretical approach for the Rician data analysis and processing.

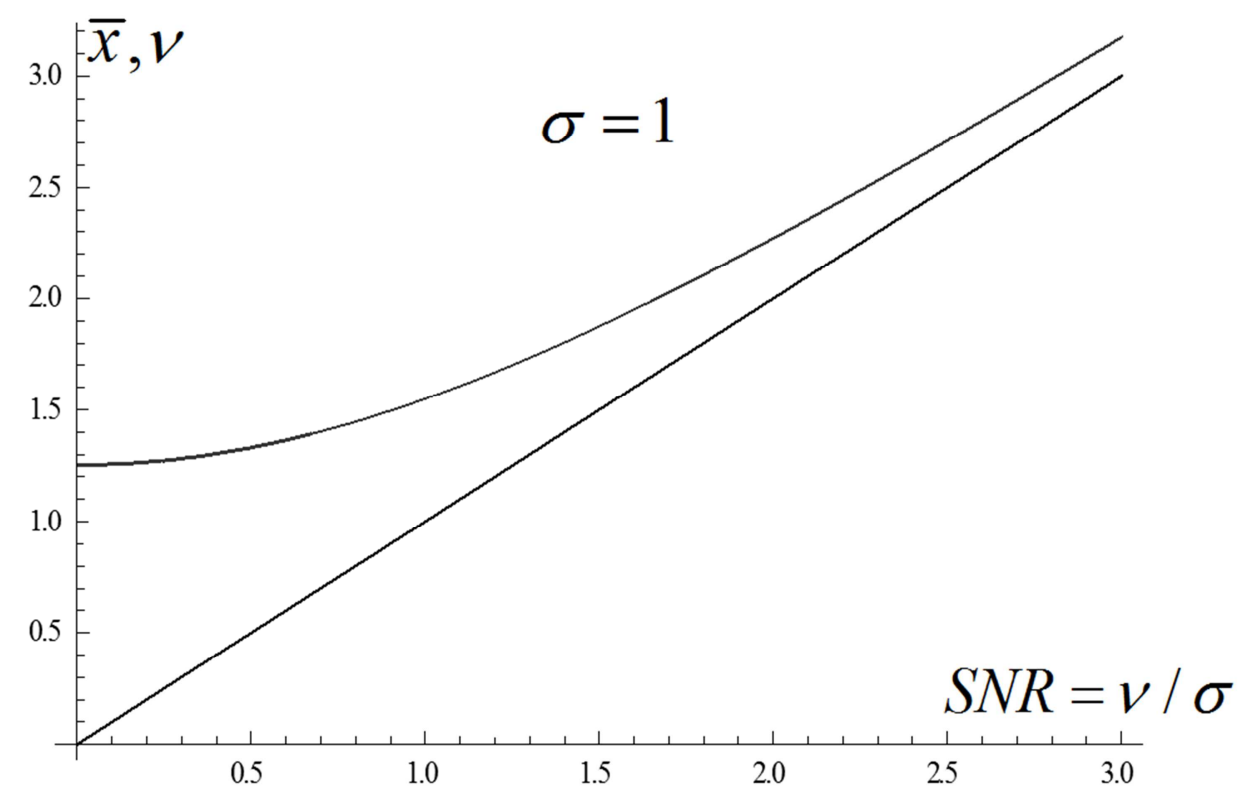

Figure 1. Illustration of the non-coincidence of the Rician signal's averaged value $\bar{x}$ and Rician parameter $v$ as dependent on the signal-to-noise ratio SNR.

In a limiting case of very high signal-to-noise ratio the Rician signal's dispersion coincides with the Gaussian noise dispersion: $\sigma_{x}^{2} \underset{v / \sigma \rightarrow \infty}{\rightarrow} \sigma^{2}$, what is natural taking into account the interconnection of the Rician and the Gaussian distributions: at $v / \sigma \gg 1$ the Rician distribution is being transformed into the Gaussian distribution with the corresponding values of parameters $v$ and $\sigma$.

In the opposite limiting case $v / \sigma \ll 1$, when the value of the useful signal is much less than the noise value, one can get $\sigma_{x}^{2} \underset{v / \sigma \ll 1}{\rightarrow} \sigma^{2} \cdot\left(2-\frac{\pi}{2}\right)$, what coincides with the known formula for the random variable with the Rayleigh distribution, what is not unexpected as at $v / \sigma \ll 1$ the Rice distribution is being transformed into the Rayleigh distribution.

Due to above indicated peculiarities of the Rice distribution the reconstruction of the initial, un-noised signal against the noise background is possible only by means of estimation of the both Rician parameters. 


\section{Substantiation of the Maximum Likelihood Technique Applicability for the Rician Data Analysis}

As mentioned above at the Rician data analysis the magnitude $x=\sqrt{x_{\mathrm{Re}}^{2}+x_{\mathrm{Im}}^{2}}$ to be measured is the modulus of the complex value with the independent real $x_{\operatorname{Re}}$ and imaginary $x_{\text {Im }}$ parts being distorted by the Gaussian noise. Let us consider a sample of $n$ measurements of the signal's amplitude $x$. The various types of the signal's averaging are denoted by the angular brackets: $\left\langle x^{k}\right\rangle=\frac{1}{n} \sum_{i=1}^{n} x_{i}^{k}$, while the average value at infinite large sample's length is denoted by the over-bar:

$$
\overline{x^{k}}=\lim _{n \rightarrow \infty} \frac{1}{n} \sum_{i=1}^{n} x_{i}^{k}
$$

The mathematical task of the Rician data two-parameter analysis consists in computing the two mentioned parameters $v$ and $\sigma^{2}$ on the basis of the sampled measurements' data.

In the present paper the maximum likelihood technique is to be substantiated as an appropriate tool for solving this problem. This means the necessity to prove that the maximum likelihood solution exists and is a unique one.

The likelihood function $L\left(v, \sigma^{2}\right)$ is expressed as a product of the probability density functions for each measurement $x_{i}$ $(i=1, \ldots, n)$ in the sample:

$$
L\left(v, \sigma^{2}\right)=\prod_{i=1}^{n} P\left(x_{i} \mid v, \sigma^{2}\right)
$$

where function $P\left(x_{i} \mid v, \sigma^{2}\right)$ is defined by (1). At the known measured sampled data function (4) is the function of unknown statistical parameters $v$ and $\sigma^{2}$. The maximum likelihood method consists in calculating those values of parameters $v$ and $\sigma^{2}$, that maximize the likelihood function $L\left(v, \sigma^{2}\right)$, or, what is equivalent, maximize its logarithm. Taking into account (1) and (4) results in the following:

$$
\begin{aligned}
& \ln L\left(v, \sigma^{2}\right)=\sum_{i=1}^{n} \ln P\left(x_{i} \mid v, \sigma^{2}\right)= \\
& \sum_{i=1}^{n}\left\{\ln x_{i}-\ln \sigma^{2}-\frac{x_{i}^{2}+v^{2}}{2 \cdot \sigma^{2}}+\ln I_{0}\left(\frac{x_{i} v}{\sigma^{2}}\right)\right\} .
\end{aligned}
$$

The maximum likelihood equations' system for calculating unknown parameters $v$ and $\sigma^{2}$ can be obtained by differentiating the logarithmic likelihood function and setting equal to zero its partial derivatives by the both sought-for parameters. Having implemented the differentiation and some mathematical treatment one can get the following system of equations for the sought for parameters $v$ and $\sigma^{2},[12,13$, 15]:

$$
\left\{\begin{array}{l}
\nu-\frac{1}{n} \sum_{i=1}^{n} x_{i} \cdot \tilde{I}\left(\frac{x_{i} v}{\sigma^{2}}\right)=0 \\
\sigma^{2}-\frac{1}{2 \cdot n} \sum_{i=1}^{n}\left(x_{i}^{2}+v^{2}\right)+\frac{v}{n} \sum_{i=1}^{n} x_{i} \cdot \tilde{I}\left(\frac{x_{i} v}{\sigma^{2}}\right)=0 .
\end{array}\right.
$$

where $\tilde{I}(z)=\frac{d}{d z} \ln I_{0}(z)=\frac{I_{1}(z)}{I_{0}(z)}$.

So the two-parameter task implies solving the system (6) of two essentially nonlinear equation with two unknown variables $v$ and $\sigma^{2}$. The numerical solution of this system in its initial view is connected with the necessity of the optimization by two parameters simultaneously, what significantly complicates the computing algorithm elaboration and increases the volume of calculations.

Obviously, the properties of the above introduced function $\tilde{I}(z)$ determine the existence of the maximum likelihood equation's solutions, their quantity and the peculiarities. The properties of this function have been studied in detail strictly proved. Here some of them are presented:

1. Function $\tilde{I}(z)$ satisfies to the following differential equation: $\frac{d}{d z} \tilde{I}(z)=1-\frac{1}{z} \cdot \tilde{I}(z)-\tilde{I}^{2}(z)$.

2. Function $\tilde{I}(z)$ is monotonically increasing on the interval $(0,+\infty)$.

3. Function $\tilde{I}(z)$ is an upward-convex function.

Taking into account these properties of function $\tilde{I}(z)$ and having implemented some mathematical treatment allow to get the following simplified system (6):

$$
\left\{\begin{array}{l}
v=\frac{1}{n} \sum_{i=1}^{n} x_{i} \cdot \tilde{I}\left(\frac{2 \cdot x_{i} \cdot v}{\left\langle x^{2}\right\rangle-v^{2}}\right) \\
\sigma^{2}=\frac{1}{2} \cdot\left(\left\langle x^{2}\right\rangle-v^{2}\right)
\end{array}\right.
$$

It is important that the first equation of system (7) - the equation for $v-$ is the one variable equation. Hence the task of solving the system of two nonlinear equations (6) for two variables $v$ and $\sigma$ has been reduced to the task of solving just one equation (7) for only one variable $v$.

Let us consider now the issue on the existence and uniqueness of the solution of the maximum likelihood equations' system (7) for the sough-for parameters $v$ and $\sigma^{2}$.

Theorem

Solution of the maximum likelihood equations' system (7) 
for parameters $v$ and $\sigma$ exists and is a unique.

Proof

From (7) it obviously follows that at existing the unique solution for $v$ it is imperative that solution for the second parameter $\sigma$ also exists and is a unique. That's why just the first equation of system (7) (equation for $v$ ) is to be considered here. The left-hand side of this equation is depicted by a straight like $y_{l}(v)=v$, while the right-hand side is a linear combinations of the functions $\tilde{I}=I_{1} / I_{0}$ :

$$
y_{r}(v)=\frac{1}{n} \sum_{i=1}^{n} x_{i} \cdot \tilde{I}\left(\frac{2 \cdot x_{i} \cdot v}{\left\langle x^{2}\right\rangle-v^{2}}\right)
$$

while the argument of function $\tilde{I}$ in each summand of the linear combination is a nonlinear function of $v$.

One can see that the value $\left(\left\langle x^{2}\right\rangle-v^{2}\right) / 2$ corresponds to the magnitude of the signal's dispersion and consequently changes insignificantly as a function of parameter $v$ because the dispersion is determined first of all by the physical nature of the noise.

Let us consider the behavior of the argument of function $\tilde{I}$ in (8) in dependence of parameter $v$. For this a denotation is introduced:

$$
g(v)=\frac{2\langle x\rangle v}{\left\langle x^{2}\right\rangle-v^{2}}
$$

Then the right-hand and the left-hand sides of the first equation of (7) can be presented as functions of a new variable $g$. This variable can be shown to be a single valued function $g(v)$ of parameter $v$ in the interval of its physically meaningful positive values.

Then the first equation of (7) can be re-written as follows:

$$
\xi(g)=\frac{1}{n} \sum_{i=1}^{n} x_{i} \tilde{I}\left(\frac{x_{i}}{\langle x\rangle} g\right)
$$

where the left-hand side is a magnitude of $v$ as a reciprocal function of argument $g: v=\xi(g(v))$.

To analyze the properties of function $g(v)$ one can get from (9):

$$
g^{\prime}(v)=2 \cdot\langle x\rangle \frac{\left\langle x^{2}\right\rangle+v^{2}}{\left(\left\langle x^{2}\right\rangle-v^{2}\right)^{2}}>0 .
$$

The positivity of derivative $g^{\prime}(v)$ means the monotonically increasing character of function $g(v)$ and thus the unambiguity of function $g(v)$ and the reciprocal function $v=\xi(g)$. Having proved the existence and the uniqueness of the non-zero solution of equation (10) for variable $g$ means thereby proving the existence and the uniqueness of the non-zero solution for parameter $v$.

Based on (9) one can obtain an analytical expression for the reciprocal function $v=\xi(g(v))$, taking into account the positivity of the physically meaningful value of parameter $v$ :

$$
v(g) \equiv \xi(g)=-\frac{\langle x\rangle}{g}+\sqrt{\left\langle x^{2}\right\rangle+\frac{\langle x\rangle^{2}}{g^{2}}} .
$$

Having analyzed the second derivative of function $v=\xi(g)$ it is not difficult to prove that this function is an upward-convex function.

The existence and the uniqueness of the solution of equation (10) are obviously determined by the presence and the quantity of the points of intersection of the curves depicting the right-hand side and the left-hand side of (10).

For the convenience of the further analysis let us denote the right-hand and the left-hand sides of equation (10) correspondingly:

$$
\begin{gathered}
\psi_{r}(g)=\frac{1}{n} \sum_{i=1}^{n} x_{i} \tilde{I}\left(\frac{x_{i}}{\langle x\rangle} g\right), \\
\psi_{l}(g)=\xi(g) .
\end{gathered}
$$

The right-hand side $\psi_{r}(g)$ of equation (10), as mentioned above, is a liner combination of monotonically increasing and upward-convex function $\tilde{I}\left(\frac{x_{i}}{\langle x\rangle} g\right)$ and consequently also is a monotonically increasing and upward-convex function that in virtue of the properties of function $\tilde{I}$ asymptotically approaches to value $v_{r}=\langle x\rangle=\frac{1}{n} \sum_{i=1}^{n} x_{i}$.

The left-hand side of equation (10) also presents a monotonous and upward-convex function $\psi_{l}(g)$,), that is analytically defined by formula (12) and asymptotically approaches to value $v_{l}=\sqrt{\left\langle x^{2}\right\rangle}$.

Thus the both sides of equation (10) are monotonically increasing convex function outgoing from the origin of coordinates. These functions asymptotically approach to horizontal lines $v=v_{r}$ and $v=v_{l}$ correspondingly. For any stochastic process the condition $\left\langle x^{2}\right\rangle>(\langle x\rangle)^{2}$ always takes place. This condition means that the asymptotic value $v_{r}$ of the right-hand side $\psi_{r}(g)$ of equation (10) is less that the asymptotic value $v_{l}$ of its left-hand side $\psi_{l}(g)$.

So, the right-hand and the left-hand sides of equation (10) are depicted by the upward-convex curves outgoing from the origin of coordinates and having different asymptotes. It is obvious that these two monotonically increasing convex 
curves may intersect only in the case if in the vicinity of zero point $g \rightarrow 0$ the derivative of the equation's right-hand side $\frac{d \psi_{r}(g)}{d g}$ exceeds the derivative of its left-hand side $\frac{d \psi_{l}(g)}{d g}$.

Having conducted the non-complicated transformations with account of above formulas one can obtain

$$
\lim _{g \rightarrow 0}\left(\frac{d \psi_{l}(g)}{d g}\right)=\frac{\left\langle x^{2}\right\rangle}{2 \cdot\langle x\rangle} \cdot\left(1-3 \cdot \frac{v^{2}}{\left\langle x^{2}\right\rangle}\right) .
$$

Similarly for the derivative of the right-hand side of (10) in the vicinity of zero point one can obtain

$$
\lim _{g \rightarrow 0}\left(\frac{d \psi_{r}(g)}{d g}\right)=\frac{\left\langle x^{2}\right\rangle}{2 \cdot\langle x\rangle}\left\{1-\frac{3}{2} v^{2} \frac{\left\langle x^{4}\right\rangle}{\left(\left\langle x^{2}\right\rangle\right)^{3}}\right\}
$$

Expressions (15) and (16) are written with the accuracy of the series expansion terms of order $v^{2}$. Calculating the difference of magnitudes (15) and (16) results in

$$
\begin{aligned}
& \lim _{g \rightarrow 0}\left\{\frac{d \psi_{r}(g)}{d g}-\frac{d \psi_{l}(g)}{d g}\right\}= \\
& =\frac{\left\langle x^{2}\right\rangle}{2\langle x\rangle} \cdot \frac{3 v^{2}}{\left\langle x^{2}\right\rangle}\left\{1-\frac{\left\langle x^{4}\right\rangle}{2\left(\left\langle x^{2}\right\rangle\right)^{2}}\right\} .
\end{aligned}
$$

Thus, the presence of the point of corresponding lines intersection, i.e. the existence of the solution of (10), depends upon the sign of the following magnitude:

$$
\beta=1-\frac{\left\langle x^{4}\right\rangle}{2 \cdot\left(\left\langle x^{2}\right\rangle\right)^{2}}
$$

where $\left\langle x^{4}\right\rangle=\frac{1}{n} \sum_{i=1}^{n} x_{i}^{4},\left\langle x^{2}\right\rangle=\frac{1}{n} \sum_{i=1}^{n} x_{i}^{2}$.

Taking into account the known formulas for the moments of the Rician variable, [17], leads to the following expression:

$$
\lim _{n \rightarrow \infty} \beta=1-\frac{8 \sigma^{4}+8 \sigma^{2} v^{2}+v^{4}}{2 \cdot\left(4 \sigma^{4}+4 \sigma^{2} v^{2}+v^{4}\right)}>0 .
$$

This means that with the growth sample length $n$ there exists such a sample length at exceeding of which condition $\beta>0$ always takes place. This means the inevitable intersection of the curves $\psi_{l}(g)$ and $\psi_{r}(g)$. The considered curves' intersection point, in virtue of the above substantiated smoothness, monotonous character and upward-convexity of both curves and with account of their radii of curvature, may be only a single one, i.e. the non-zero solution of equation (10) and exists and is a unique. The proved existence and uniqueness of the solution for parameter $v$ obviously means the existence and the uniqueness of the solution for the second parameter $\sigma$.

Thus, the solution of equations' system (7) exists and is a unique. The theorem is proved.

The solution of the equations system (7), obtained by setting the derivative of the likelihood function equal to zero, defines the extremum point of the likelihood function that may correspond to both the maximum and the minimum. To determine the character of extremum the sign of the second derivative of the logarithmic likelihood function $\frac{\partial^{2}}{\partial v^{2}} \ln L\left(v, \sigma^{2}\right)$ has been investigated. As its rather lengthy mathematical analysis cannot be presented within the frameworks of the present paper, just the resultant conclusion is provided here: at the presence of a non-trivial solution $v \neq 0$ the second derivative of the logarithmic likelihood function is positive at $v \rightarrow 0$. This means that at existence of the non-trivial solution of system (7) for parameter $v$ the trivial solution $v=0$ corresponds to just a minimum, not a maximum of the likelihood function. By the other words the trivial solution in this case is not a sought for solution of the maximum likelihood method. So, just the non-trivial solution $v \neq 0$ of the equations system (7) always corresponds to the likelihood function's maximum.

The situation, when the useful signal is absent $(v=0)$, corresponds to the Rayleigh distribution as a particular case of the Rice distribution, and in this case the only solution of the equation for parameter $v$ of system (7) is a trivial solution $v=0$. With account of the series expansion of function $\tilde{I}(z)$ at small values of the argument, one can get the following expression for the second derivative of the logarithmic likelihood function $\ln L_{R}\left(v, \sigma^{2}\right)$ for the Rayleigh distribution:

$$
\frac{\partial^{2}}{\partial v^{2}} \ln L_{R}\left(v, \sigma^{2}\right)=-\frac{3}{8} \cdot \frac{\left\langle x^{4}\right\rangle}{\left\langle x^{2}\right\rangle^{2}} \cdot v^{2}<0 .
$$

The negative value of the second derivative (20) means that the single extremum of the likelihood function, corresponding to the trivial solution $v=0$ is a maximum of this function.

\section{The Stable Character of the Rice Statistical Distribution}

If a sum of two independent random variables obeying to the same distribution (probably, of different parameters) also obeys to the same distribution then such a distribution is said to be a stable one. This section considers the issue of the Rice distribution's stable character. To prove it let us present the Rician signal as a sum of the determined signal and the Gaussian noise, as illustrated by Figure 2.

Taking into account that the noise amplitude $r$ obeys to the Rayleigh distribution while the noise phase $\psi$ is distributed uniformly in interval $(0,2 \pi)$, one can obtain for the joint 
probability density function $u_{R}(R, \varphi)$ for the resulting vector's amplitude $R$ and phase $\varphi$ the following formula:

$$
\begin{aligned}
& u_{R}(R, \varphi) d R d \varphi= \\
& \exp \left\{-\frac{R^{2}+A^{2}-2 R A \cos \left(\varphi-\varphi_{0}\right)}{2 \sigma^{2}}\right\} \frac{R d R d \varphi}{2 \pi \sigma^{2}}
\end{aligned}
$$

To prove the stable character of the Rice distribution let us consider a distribution of a signal having been formed by summing of two Rician signals. In practice usually the situation takes place when the signals being summed are characterized by an amplitude and a phase. So, these signals are represented as vectors while the case of the scalar adding is just a particular case of the equal phases of the signals to be added.

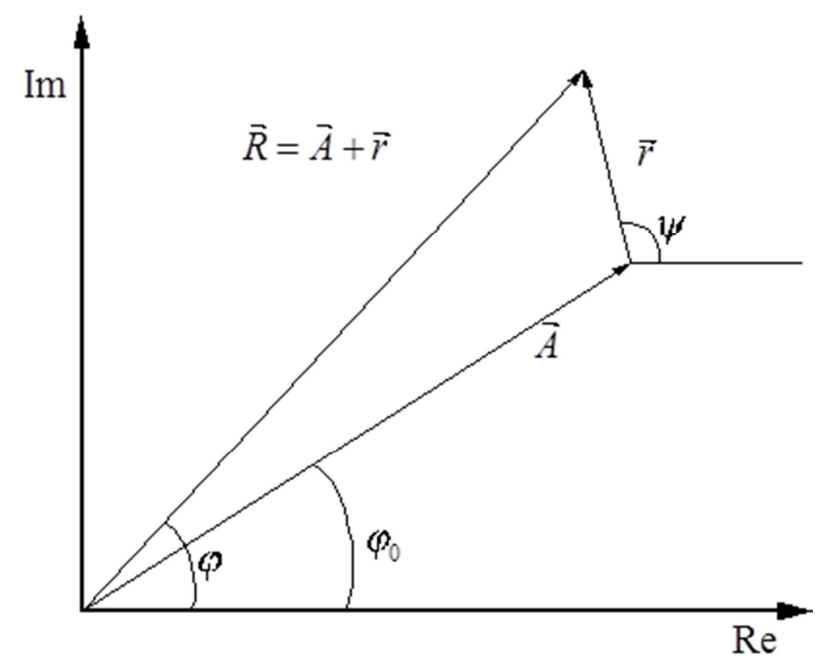

Figure 2. Illustration of formingthe Ricisn signal $R=|\vec{R}|$ as a result of the impact of Gaussian noise $\vec{r}$ onto the initially determined signal of amplitude $A$.

Let vectors $\vec{R}_{1}$ and $\vec{R}_{2}$ denote two initial signals with the amplitudes $R_{1}$ and $R_{2}$ obeying to the Rice distribution with parameters $A_{1}, \sigma_{1}$, and $A_{2}, \sigma_{2}$, correspondingly. Let $\beta_{1}$ and $\beta_{2}$ be the phases of the corresponding noise components of two signals to be added. So, phases $\beta_{1}$ and $\beta_{2}$ are uniformly distributed in interval $(0,2 \pi)$. This is illustrated by Figure 3.

The components of the sum vector $\vec{R}=\vec{R}_{1}+\vec{R}_{2}$ are as follows:

$$
\begin{aligned}
& R_{x}=R_{1} \cos \varphi_{1}+R_{2} \cos \varphi_{2}= \\
& A_{1} \cos \varphi_{10}+r_{1} \cos \beta_{1}+A_{2} \cos \left(\varphi_{10}+\Delta \varphi\right)+r_{2} \cos \beta_{2}, \\
& R_{y}=R_{1} \sin \varphi_{1}+R_{2} \sin \varphi_{2}= \\
& A_{1} \sin \varphi_{10}+r_{1} \sin \beta_{1}+A_{2} \sin \left(\varphi_{10}+\Delta \varphi\right)+r_{2} \sin \beta_{2} .
\end{aligned}
$$

where $\varphi_{10}$ and $\varphi_{20}=\varphi_{10}+\Delta \varphi$ - the phases of vectors $\vec{R}_{1}$ and $\vec{R}_{2}$, correspondingly.

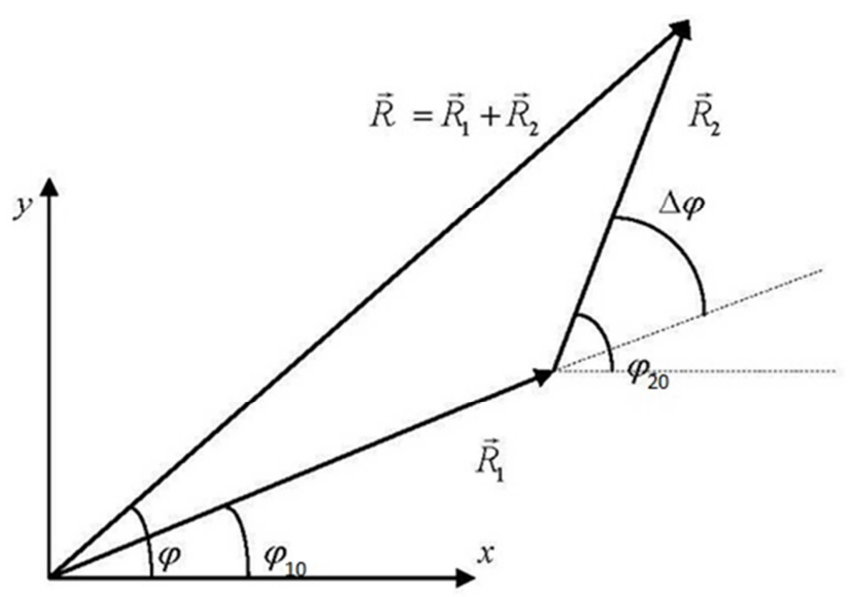

Figure 3. Illustrationto the proof of the Rice distribution's stable character.

The normal distribution is known to possess a quality of unlimited decomposability what means that any normally distributed random variable can be presented as a sum of an arbitrary number of normal random variables. Due to this fact it is not difficult to see that amplitude $R$ of signal $\vec{R}=\vec{R}_{1}+\vec{R}_{2}$ obeys to the Rice distribution (as well as amplitudes $R_{1}$ and $R_{2}$ ), because the components of its noise vector $\vec{r}=\vec{r}_{1}+\vec{r}_{2}$ are the normally distributed random variables. The next task being solved below consists in the determination of the Rician parameters for amplitude $R$ of the resultant vector $\vec{R}=\vec{R}_{1}+\vec{R}_{2}$.

The components $R_{x}$ and $R_{y}$ can be presented as follows:

$$
\left\{\begin{array}{l}
R_{x}=R_{x 0}+r \cos \beta \\
R_{y}=R_{y 0}+r \sin \beta
\end{array}\right.
$$

where

$$
\begin{aligned}
& R_{x 0}=A_{1} \cos \varphi_{10}+A_{2} \cos \left(\varphi_{10}+\Delta \varphi\right) \\
& R_{y 0}=A_{1} \sin \varphi_{10}+A_{2} \sin \left(\varphi_{10}+\Delta \varphi\right)
\end{aligned}
$$

On the other hand one can obviously put down $R_{x}$ and $R_{y}$ through amplitude $R$ and phase $\varphi_{\text {of the resulting vector }}$ $\vec{R}$ :

$$
\begin{aligned}
& R_{x}=R \cos \varphi \\
& R_{y}=R \sin \varphi
\end{aligned}
$$

From $(23)-(25)$ it follows:

$$
\begin{aligned}
& r \cos \beta=R \cos \varphi- \\
& -\left[A_{1} \cos \varphi_{10}+A_{2} \cos \left(\varphi_{10}+\Delta \varphi\right)\right] \\
& r \sin \beta=R \sin \varphi- \\
& -\left[A_{1} \sin \varphi_{10}+A_{2} \sin \left(\varphi_{10}+\Delta \varphi\right)\right]
\end{aligned}
$$

The values in the square brackets are the predetermined, not random ones. 
Taking into account the Rayleigh distribution of amplitude $r$ of the noise vector $\vec{r}$ and the uniform distribution of its phase $\beta$ one can get:

$$
u_{r} d r=\frac{e^{-r^{2} / 2 \sigma^{2}}}{\sigma^{2}} r d r
$$

where $\sigma^{2}=\sigma_{1}^{2}+\sigma_{2}^{2}$. Then, from (26) as a result of non-complicated mathematical transformation it follows:

$$
\begin{aligned}
& r^{2}=R^{2}+A_{1}^{2}+A_{2}^{2}+2 A_{1} A_{2} \cos \Delta \varphi- \\
& -2 R\left[A_{1} \cos \left(\varphi-\varphi_{10}\right)+A_{2} \cos \left(\varphi-\varphi_{20}\right)\right]
\end{aligned}
$$

Taking (28) into account and having conducted some mathematical treatment one can put down the joint probability density function $u_{R}(R, \varphi)$ foramplitude $R$ and phase $\varphi$ of the summand signal as follows:

$$
\begin{aligned}
& \tilde{u}_{R}(R, \varphi) d R d \varphi= \\
& =\exp \left\{-\frac{R^{2}+A_{0}^{2}-2 R A_{0} \cos (\varphi-\delta)}{2 \sigma^{2}}\right\} \frac{R d R d \varphi}{2 \pi \sigma^{2}}
\end{aligned}
$$

where

$$
\begin{aligned}
& A_{0}=\sqrt{A_{1}^{2}+A_{2}^{2}+2 A_{1} A_{2} \cos \Delta \varphi} \\
& \delta=\varphi_{0}+\operatorname{arctg}\left[\frac{\left(A_{2}-A_{1}\right)}{\left(A_{2}+A_{1}\right)} \operatorname{tg} \frac{\Delta \varphi}{2}\right]
\end{aligned}
$$

In (30) $A_{0}$ is the amplitude of resulting signal as calculated at the absence of a noise, and $\delta$ - an angle value corresponding to the un-noised resulting signal.

Comparing (21) and (39) provides a strict proof of the fact that the sum signal obeys to the same distribution for its amplitude and phase as a distribution of each of the summand signals.

Integrating (29) by phase variable $\varphi$ allows getting the following expression for the probability density function for the resultant signal's amplitude $R$ :

$$
\omega_{R}(R) d R=I_{0}\left(\frac{R A_{0}}{\sigma^{2}}\right) e^{-\left(R^{2}+A_{0}^{2}\right) / 2 \sigma^{2}} \frac{R d R}{\sigma^{2}}
$$

This means that the amplitude of the resultant signal obeys to the Rice distribution with parameters $A_{0}$ and $\sigma^{2}=\sigma_{1}^{2}+\sigma_{2}^{2}$.

So, the stable character of the Rice statistical distribution is strictly proved.

\section{Conclusion}

The paper provides a detailed theoretical study of the peculiarities inherent to the Rice statistical model. The efficient reconstruction of the Rician signal against the noise background is shown to be achieved only on the basis of joint determination of both a priori unknown Rician parameters. The Rice distribution properties, such as the Rician likelihood function features and the stable character of the Rice distribution, are mathematically investigated. The uniqueness of the likelihood function maximum is strictly proved, what substantiates the applicability of the maximum likelihood technique for the Rican data analysis. Besides, the Rice distribution is shown to be a stable one, what makes it possible to apply the Rice model in many tasks when the signal being analyzed is formed as a sum of the Rician signals. The investigated features of the Rice statistical distribution allow efficient solving the task of calculation of the both Rician parameters which is of special importance at data processing as it is directly connected with solving the problem of the separation of the informative and the noise components of the analyzed data.

\section{Acknowledgements}

The work has been supported by RFBR, project N17-07-00064 within the Fundamental Research program.

\section{References}

[1] S. O. Rice, "Mathematical Analysis of Random Noise", Bell Syst. Tech. Journal, 1944. Vol. 23. p. 282-322.

[2] T. R. Benedict, T. T. Soong, "The joint estimation of signal and noise from the sum envelope", IEEE Trans. Inf. Theory, 1967. Vol. IT-13. No. 3. p. 447-454.

[3] K. K. Talukdar, W. D. Lawing, "Estimation of the parameters of Rice distribution", J. Acoust. Soc. Amer., 1991. Vol. 89. No. 3. p. 1193-1197.

[4] J. Sijbers, A. J. den Dekker, P. Scheunders, D. VanDyck, "Maximum-Likelihood Estimation of Rician Distribution Parameters", IEEE Transactions on Medical Imaging, 1998. Vol. 17. No 3. p. 357-361.

[5] T. V. Yakovleva, "Conditions of Rice statistical model applicability and estimation of the Rician signal's parameters by maximum likelihood technique", Computer Research and Modeling, 2014, vol. 6, no. 1, pp. 13-25.

[6] T. M. Cover, J. A. Thomas Elements of Information Theory. John Wiley and Sons, 2006. 776p.

[7] A. J. den Dekker, J. Sijbers "Data distributions in magnetic resonance images: A review", Physica Medica: European Journal of Medical Physics, 2014. Vol. 30. Issue 7. p. 723-741.

[8] T. V. Yakovleva, "A Theory of Signal Processing at the Rice Distribution”, Dorodnicyn Computing Centre, RAS, Moscow, 2015. - 268p.

[9] C. F. M. Carobbi, M. Cati, L. M. Millanta, “A New Procedure for Evaluating the Performance of the Site for Radiation Test or Antenna Calibration”, EMC Europe 2004, International Symposium on Electromagnetic Compatibility, Symposium Record, Eindhoven, The Netherlands, 2004. Vol. 2. p. $702-706$. 
[10] T. V. Yakovleva, A. V. Kniazkov, "Speckle-noise computing by two-parameter analysis of the reflected light's periodic variation", Optical Memory \& Neural Networks (Information Optics), 2014. Vol. 23. №4. p. 233-241.

[11] T. V. Yakovleva, A. V. Kniazkov, "Comparison of Two Techniques of Electro-Optical Coefficient Evaluation", Physics Procedia, 2015. Vol. 73, p. $189-192$.

[12] T. V. Yakovleva, N. S. Kulberg, "Special features of the Likelihood Function of the Rice Statistical Distribution", Doklady Mathematics, 2014. Vol. 90. No. 1. p. 472-475.

[13] T. V. Yakovleva, N. S. Kulberg, "Mathematical Statistics Methods as a Tool of Two-Parameter Magnetic-Resonance Image Analysis", Informatics and its application, 2014. v. 8. is. 3. p. 79-89.
[14] T. V. Yakovleva, N. S. Kulberg, "Methods of Mathematical Statistics in Two-Parameter Analysis of Rician Signals", Doklady Mathematics. 2014. Vol. 90, No. 3. P. 1-5.

[15] Tatiana V. Yakovleva, Nicolas S. Kulberg, "Noise and Signal Estimation in MRI: Two-Parametric Analysis of Rice-Distributed Data by Means of the Maximum Likelihood Approach", American Journal of Theoretical and Applied Statistics, 2013. Vol. 2. No. 3. p. 67-79.

[16] M. Abramovits, I. Stegun, Handbook of Mathematical Functions, M.:Nauka, 1979. 832 p.

[17] J. H. Park, Jr., "Moments of generalized Rayleigh distribution", Q. Appl. Math., 1961. Vol. 19. No. 1. p. 45-49. 